\title{
Investigation of Enhanced Ion Abundances from a Carrier Process in High-Performance Liquid Chromatography Particle Beam Mass Spectrometry
}

\author{
Thomas A. Bellar, Thomas D. Behymer, and William L. Budde \\ U.S. Environmental Protection Agency, Office of Research and Development, Environmental \\ Monitoring Systems Laboratory, Cincinnati, Ohio, USA
}

\begin{abstract}
Enhancements in ion abundances in high-performance liquid chromatography (HPLC) particle beam (PB) mass spectrometry were observed during coelution or when certain substances (e.g., ammonium acetate) were added to the mobile phase during a reverse-phase gradient elution HPLC separation. These enhancements were observed with two commercial PB interfaces and a variety of compounds generally not amenable to separation by gas chromatography. The enhancements are attributed to both improved chromatographic efficiency and a PB carrier process. (J Am Soc Mass Spectrom 1990, 1, 92-98)
\end{abstract}

$\mathrm{P}$ article beam (PB) interfaces for high-performance liquid chromatography (HPLC)/mass spectrometry systems have been described [1-3], but very limited information is available concerning their performance characteristics with a variety of analytes and liquid chromatographic conditions. During our investigations on a test mixture of compounds, we noted enhanced positive ion abundances for some polar compounds when ammonium acetate was added to the mobile phase. Similarly, enhanced ion abundances were observed for coeluting compounds, and this enhancement could be modified to a certain extent in a potentially useful way by the addition of ammonium acetate.

While some of the effects of ammonium acetate are the result of more favorable LC conditions, a significant fraction of the enhanced positive ion abundances is associated with a PB carrier process. The purpose of this work is to provide a quantitative description of the ion abundance enhancements and to provide data to support the concept of a carrier process. These observations are significant in that additives, such as ammonium acetate, may be used not only to improve chromatographic behavior but also to increase analyte signal intensity and therefore lower the instrument detection limits. But this carrier process also has negative implications in regard to the potential for quantitative analyses using $\mathrm{PB} / \mathrm{HPLC} / \mathrm{MS}$, particularly with coeluting stable-isotope-labeled internal standards.

Address reprint requests to William L. Budde, USEPA-Cincinnati, Environmental Monitoring Systems Laboratory, $26 \mathrm{~W}$. Martin Luther King Drive, Cincinnati, OH 45268, USA.

\section{Experimental}

\section{Materials}

Compounds used in this work were the highest purity materials available from commercial sources or from the U.S. Environmental Protection Agency's (USEPA) Repository for Toxic and Hazardous Materials and were used without purification. Acetonitrile was Burdick and Jackson UV grade, and reagent water was obtained from a Milli-Q (Millipore Corp.) water purification system. Ammonium acetate was ACS reagent grade. High-purity helium was obtained from the U.S. Bureau of Mines.

\section{Instrumentation}

Two PB/HPLC/MS systems were used in this work. System A was a Hewlett-Packard system consisting of a Model 1090 high-performance liquid chromatograph, a 59980A PB interface, and a Model 5988 quadrupole mass spectrometer with a high-energy dynode detector and an RTE/A data system. The interface consisted of standard components except that the plastic beam collimator was replaced with an aluminum part provided by the manufacturer. System B consisted of a Waters PB 600 MS high-performance liquid chromatograph, an Extrel Thermabeam interface, and an Extrel ELQ 400-1 quadrupole mass spectrometer with an Extrel RSX11M-based data system. Other equipment and most operating conditions were the same on both systems, and all HPLC injections were made with autoinjectors. Postcolumn addition was accomplished with a Waters Model 590 HPLC pump. 


\section{Liquid Chromatography}

The HPLC column was a Waters Bondapack $30 \mathrm{~cm} \times 2$ mm (i.d.) packed with irregular $10-\mu \mathrm{m}$ siliça particles with octadecyldimethylsilyl (C-18) groups chemically bonded ( $10 \%$ loading) to the silica surface. The mean pore size was $125 \AA(50-300 \AA)$, and some residual acidic sites were endcapped with methyl groups. Supelco LC-18 or DuPont C-18 guard columns were used to protect the analytical column. The columns were thoroughly conditioned by pumping a $50 \%$ reagent water-acetonitrile solution through them for an extended period (several hours to several days) to remove residual impurities, column bleed, and so on.

The mobile phase was a mixture of acetonitrile and reagent water or acetonitrile and reagent water containing ammorium acetate. The solvents were degassed in an ultrasonic bath under reduced pressure and continuously purged with helium at about $30 \mathrm{~mL} / \mathrm{min}$ to maintain very low levels of dissolved air. The solvent composition was held for $1 \mathrm{~min}$ at $30 \%$ acetonitrile and then linearly programmed to $100 \%$ acetonitrile in 29 min. Injection volume was $5 \mu \mathrm{L}$, and the mobile phase flow rate was either $0.40 \mathrm{~mL} / \mathrm{min}$ or, when postcolumn addition of reagent water or ammonium acetate solution was used, $0.36 \mathrm{~mL} / \mathrm{min}$. Postcolumn addition was accomplished by adding $0.04 \mathrm{~mL} / \mathrm{min}$ of either reagent water or ammonium aretate solution immediately after the HPLC column but before the PB interface. Therefore, the flow rate into the interface was the same with and without postcolumn addition.

During experiments without postcolumn addition, the ammonium acetate concentration in the mobile phase gradually decreased as the fraction of aceturitrile increased during the gradient elution. The initial ammonium acetate concentration in the reagent water solvent was usually $0.01 \mathrm{M}$. The experiments in which the initial ammonium acetate concentration in the reagent water solvent was varied from 0 to $0.05 \mathrm{M}$ were conducted without postcolumn addition. When postcolumn addition of ammonium acetate was made, a $0.1 \mathrm{M}$ solution was added so the final concentration entering the interface was $0.01 \mathrm{M}$.

\section{Particle Beam and Mass Spectrometry Conditions}

The nebulizing gas was high-purity helium, and the temperature of the outer concentric tube of the nebulizer (helium delivery tube) was ambient in system A and $105{ }^{\circ} \mathrm{C}$ in system $B$. The helium flow rate in both systems was aboul $1.5 \mathrm{~L} / \mathrm{min}$, and this was precisely controlled by a pressure regulator and a flow restrictor. The temperatures of the desolvation chambers were 45 ${ }^{\circ} \mathrm{C}$ (system $\mathrm{A}$ ) and $80^{\circ} \mathrm{C}$ (system B) as measured at the surfaces of the exterior walls.

The two-stage momentum separator rough pumps in the PB interfaces reduced the pressure from about $0.5 \mathrm{~atm}$ in the desolvation chambers to about 0.3 torr at the second-stage rough pumps. The two spectrom- eter diffusion (system A) or turbo (system B) pumps maintained a pressure of $1.5-3 \times 10^{-3}$ torr in the manifolds surrounding the conventional electron ionization (EI) ion sources. The ion source temperatures were 250 ${ }^{\circ} \mathrm{C}$, and the electron energy was $70 \mathrm{eV}$. The mass spectrometers were repetitively scanned from 62 to $450 \mathrm{u}$ at $1.5 \mathrm{~s} / \mathrm{scan}$, which usually gave at least ten scans per HPLC peak. All spectra were acquired in the positive ion mode.

\section{Ion Source Tuning and Calibration}

The ion source and lens potentials were adjusted manually to optimize ion intensity from perfluorotributylamine (PFTBA) in the 100-220-u range while a 50\% water-acetonitrile solution was pumped through the interface. Both spectrometers' mass scales were calibrated over the mass range of $62-450 u$ with PFTBA according to the manufacturer's procedures.

\section{Results and Discussion}

The compounds shown in Table 1 (test mixture) were selected for study, and their EI spectra and other aspects of their PB/HPLC/MS analyses will be described in another publication. The test mixture was chromatographed on a C-18 reverse-phase column using an acetonitrile-water gradient elution, and most components eluted to give reasonably narrow peaks (Figure 1). But benzidine, 3,3'-dimethylbenzidine, and 3,3'dimethoxybenzidine, all basic aromatic diamines, were strongly retained on the column (and on several other C-18 columns from other suppliers). These three compounds gave broad weak peaks between 11 and $15 \mathrm{~min}$ (Figure 1) and could not be detected with high sensitivity until $0.01 \mathrm{M}$ ammonium acetate was added to the mobile phase (Figure 2).

The presence of this relatively volatile salt caused earlier elution of the three benzidines as reasonably symmetrical and narrow peaks and enhanced ion abundances for all compounds in the test mixture (compare total ion abundances in Figures 1 and 2 for the same quantities injected, 100-1500 $\mathrm{ng}$ of each). Therefore, although the presence of ammonium acetate in the mobile phase was necessary for the three aromatic diamines, it also caused potentially useful ion abundance enhancements with other compounds.

With the basic diamines, improved LC (peak width and symmetry) and enhanced sensitivity from greater throughput are expected when acidic (silanol) sites on the column, which strongly retain basic compounds, are blocked by the relatively strong base ammonia. The diamine 3, $3^{\prime}$-dichlorobenzidine is less basic because of the electron-withdrawing chlorine atoms and is not as strongly bound to the acidic sites. Thus it elutes as a relatively narrow peak without ammonium acetate (Figure 1). Reasonably good chromatography is also observed with the other nonbasic compounds in the test mixture without ammonium ac- 
Table 1. Particle beam carrier enhancement factors from postcolumn addition of ammonium acetate to the mobile phase

\begin{tabular}{|c|c|c|c|c|c|c|c|}
\hline \multirow[b]{2}{*}{ Compound } & \multirow[b]{2}{*}{ Abbr. } & \multirow[b]{2}{*}{$\mathrm{MW}^{\mathrm{a}}$} & \multirow{2}{*}{$\begin{array}{c}\text { Quantization } \\
\text { ion }\end{array}$} & \multicolumn{2}{|c|}{ Amt, injected (ng) } & \multicolumn{2}{|c|}{ Mean enhancement factor ${ }^{b}$} \\
\hline & & & & Syst. A & Syst. B & Syst. A & Syst. B \\
\hline Benzidine & $\mathrm{BZ}$ & 184 & 184 & 400 & 720 & 2.5 & 3.5 \\
\hline Benzidine- $D_{8}$ & HB & 192 & 192 & 100 & 170 & 5.2 & 3.7 \\
\hline Benzoylpropethyl & BP & 365 & 105 & 400 & 720 & 1.8 & 1.2 \\
\hline Caffeine & $\mathrm{CF}$ & 194 & 194 & 180 & 330 & 1.9 & 1.7 \\
\hline Caffeine- ${ }^{-15} \mathrm{~N}_{2}$ & $\mathrm{HC}$ & 196 & 196 & 102 & 230 & 2.0 & 1.4 \\
\hline Carbaryl & $\mathrm{CL}$ & 201 & 144 & 800 & 1400 & 1.5 & 1.6 \\
\hline o-Chlorophenyl thiourea & PT & 186 & 151 & 202 & 460 & 5.1 & 1.6 \\
\hline $3,3^{\prime}$-Dichlorobenzldine & $\mathrm{DB}$ & 252 & 252 & 400 & 720 & 2.1 & 1.2 \\
\hline $3,3^{\prime}$-Dichlorobenzidine $-D_{6}$ & $H D$ & 258 & 258 & 100 & 170 & 2.7 & 1.3 \\
\hline 3,3'-Dimethoxybenzidine & MB & 244 & 244 & 450 & 810 & 5.7 & 3.8 \\
\hline 3,3'-Dimethylbenzidine & LB & 212 & 212 & 400 & 720 & 4.8 & 2.3 \\
\hline Diuron & DI & 232 & 72 & 79 & 210 & 3.7 & 1.8 \\
\hline Ethylene thiourea & ET & 102 & 102 & 400 & 720 & 1.8 & 1.7 \\
\hline Linuron & LI & 248 & 248 & 1200 & 2200 & 0.6 & 1.2 \\
\hline Rotenone & RO & 394 & 192 & 1500 & 2700 & 2.0 & 1.7 \\
\hline Siduron & SI & 232 & 93 & 308 & 660 & 4.1 & 1.6 \\
\hline
\end{tabular}

- Monoisotopic MW calculated from the atomic masses of the isotopes with the smallest atomic masses.

b Ratio of the mean integrated abundance of the quantitation ion with postcolumn addition of ammonium acetate to the mean integrated abundance with postcolumn addition of reagent water; mean of four measurements.

etate, but its presence caused enhanced ion abundances for all compounds studied (Figure 2). Another ion abundance-enhancing mechanism appeared to be operational. This was confirmed with flow injection analyses, for which the HPLC column was removed and individual analytes injected into the mobile phase and pumped directly into the PB interface.

The effect of ammonium acetate on ion abundance was studied to ascertain what processes, in addition to improved chromatographic efficiency, might be operational. Accurate measurements of ion abundance enhancements are impossible with HPLC columns that have been exposed to ammonium acetate, because residual quantities of the salt are difficult to wash out of the column packing. Therefore, enhancement measurements were conducted with unexposed columns and with postcolumn addition of ammonium acetate to separate the PB carrier process from any signal enhancement caused by improved chromatographic efficiency. Furthermore, the HPLC columns were thoroughly conditioned with reagent water-acetonitrile for extended periods (several hours to several days) to remove column bleed and residual impurities that may affect the formation of the particle beam.

Using an HPLC column that had not been exposed to ammonium acetate, the test mixture was chromatographed with an acetonitrile-reagent water mobile phase without ammonium acetate. After separation, either reagent water or ammonium acetate in reagent water was added to the effluent from the column before it entered the PB interface. Measured in this way, ion abundance enhancements are for a spe-
Figure 1. Tutal ion current profile from the acetonitrile-reagent water gradient elution of the mixture of sixteen compounds in Table 1 with postcolumn addition of reagent water.

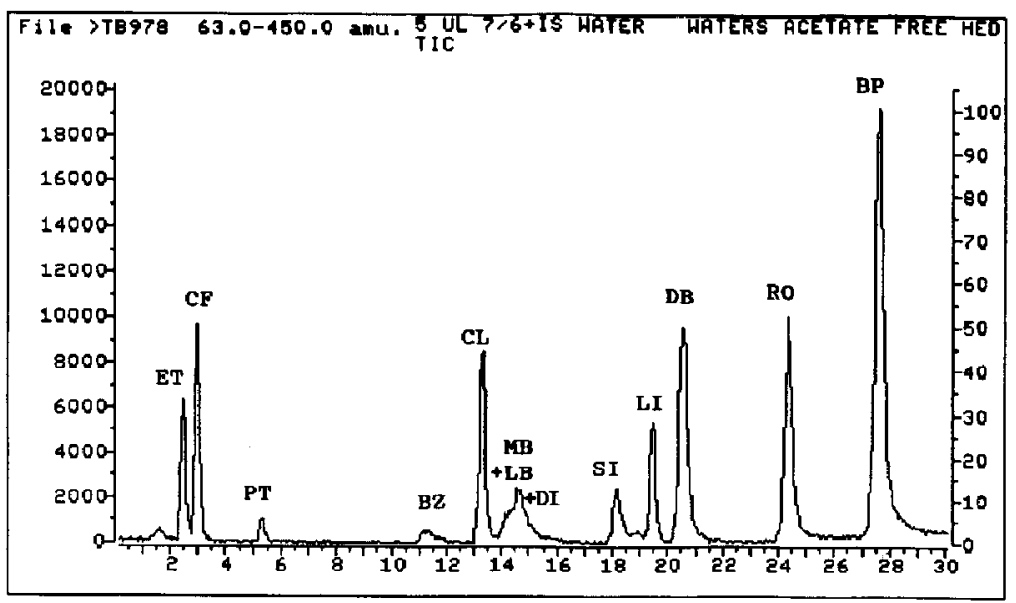




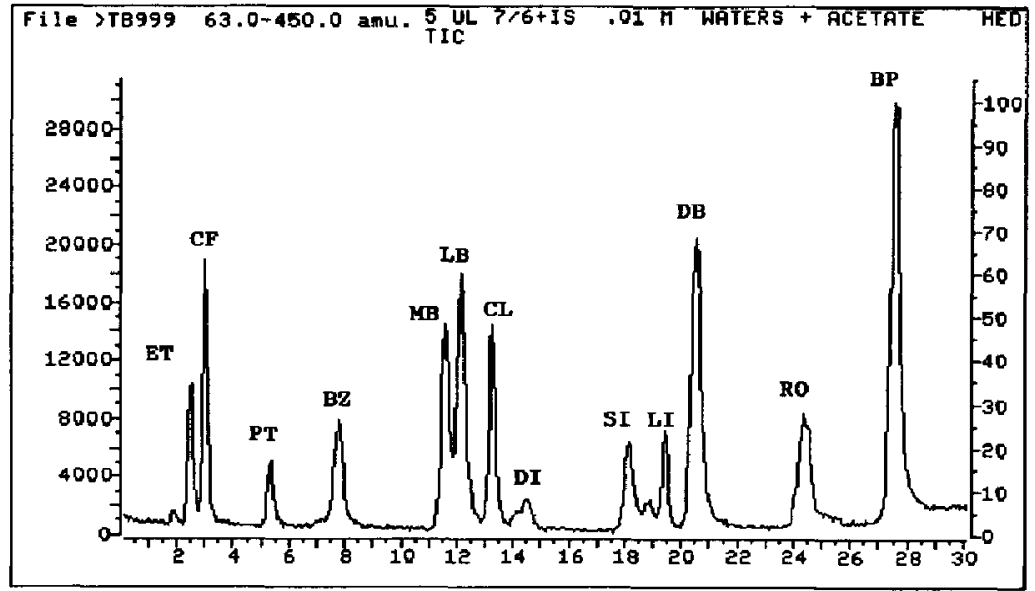

Figure 2. Total ion current profile from the acetonitrile- $0.01 \mathrm{M}$ ammonium acetate in water gradient elution of the mixture of sixteen compounds in Table 1. cific acetonitrile-water mixture that is somewhat different for each compound, because each elutes at a different point in the acetonitrile-water gradient elution. But the concentration of ammonium acetate in the interface is constant for all compounds, that is, $0.01 \mathrm{M}$. Figure 3 is a total ion chromatogram from postcolumn addition of ammonium acetate, and the three most basic aromatic diamines elute as broad peaks but give enhanced ion abundances, along with all other compounds (compare Figures 1 and 3 ).

An enhancement factor is defined as the ratio of the integrated quantitation ion abundance with postcolumn addition of ammonium acetate to the integrated abundance with postcolumn addition of reagent water. Mean enhancement factors were calculated from four separate injections of the analyte mixture with postcolumn addition of reagent water or ammonium acetate. Most coeluting or partially coeluting compounds were excluded from the test mixture to eliminate other ion abundance-enhancing effects. But three pairs of coeluting stable-isotnpe-labeled compounds were in the test mixture to determine the effects of coeluting compounds with identical structures on ion abundance. The coeluting stable-isotope-labeled compounds are not identified in Figures 1-3, but they were in the test mixture.

Table 1 shows the molecular weights (MW) of the compounds in the test mixture, the quantitation ions (usually the base peaks), the quantities of each compound injected (in ng), and the mean enhancement factors from the postcolumn addition experiments just described. In these experiments, the relative standard deviations (RSD) of the integrated quantitation ion abundances were in the range of $5-15 \%$, but an RSD greater than $25 \%$ was obtained for benzidine- $D_{8}$ in reagent water with system A. A limited quantity of this material was available (100 $\mathrm{ng}$ injected), and without ammonium acetate benzidine produces a weak signal that could not be measured precisely (Figure 1). Therefore, although most enhancement factors of corresponding native and isotope-labeled compounds measured on the same system are in reasonable agreement, the benzidine enhancement factors from system $A$ do not compare well because the henzidine- $D_{B}$ signal was less precisely measured near the instrument detection limit. Further studies of the precision of ion

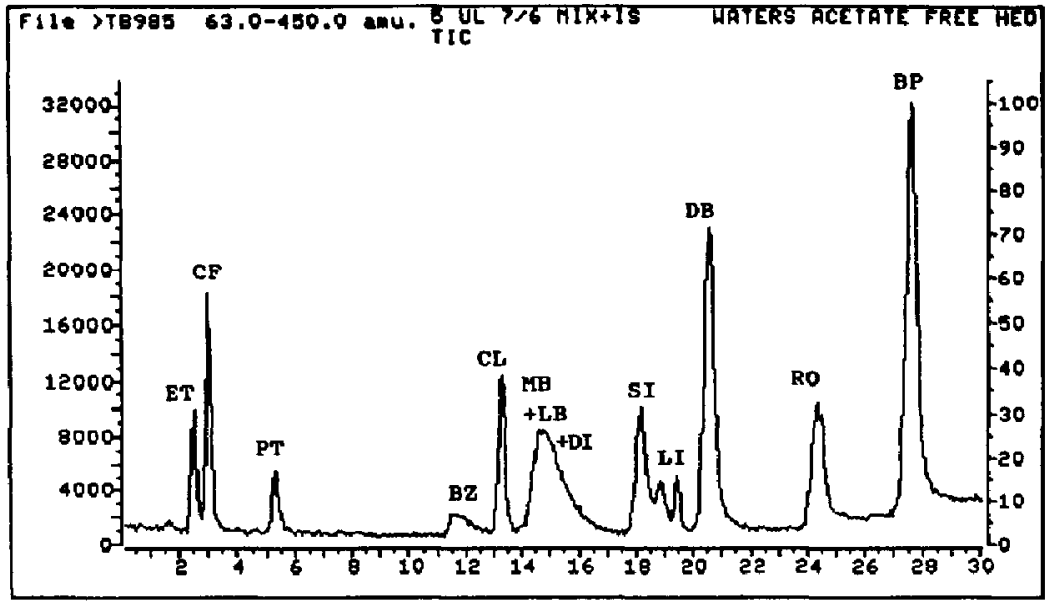

Figure 3. Total ion current profile from the acetonitrile-reagent water gradient elution of the mixture of sixteen compounds in Table 1 with postcolumn addition of ammonium acetate in water. 
abundance measurements and the effects on quantitative analyses will be published separately.

Enhancement factors from system A, which operates at ambient temperature, range from 1.5 to 5.7 except for linuron, for which a negative effect is observed. With system $B$ the outer concentric tube of the nebulizer (helium delivery tube) was at $105^{\circ} \mathrm{C}$, and the enhancement factors range from 1.2 to 3.8 with a $20 \%$ enhancement in the linuron signal. No apparent correlation exists between enhancement factors and retention times (solvent composition) or analyte structure, except that the stronger bases among the analytes, for example, 3, 3'-dimethyl- and 3, 3'-dimethoxybenzidine, generally have higher enhancement factors. Also, the enhancement factors for the three coeluting pairs (native and isotope-labeled) are not pure and include contributions from ammonium acetate and from the coeluting substances (see discussion below).

Enhancement factors that vary with structure tend to rule out the simple physical explanation that ammonium acetate promotes particle formation, which increases analyte transmission efficiency. Enhancement factors appear to depend on specific conditions such as the temperature of the nebulizing gas (higher temperatures produce a drier spray with smaller particles). A thorough study is planned on the effect of nebulizer temperature on ion abundance enhancements, and these results will be reported in a separate publication.

Because the nebulization process used in the PB systems (particularly system B) is at least analogous to that used in thermospray $[4,5]$, ions may be formed in the desolvation chambers. One possible explanation for the ammonium acetate carrier process is the formation of adduct ions $(M+1$ and $M+18)$. These adduct ions, which are more soluble in the liquid phase and less volatile than the analytes themselves, would increase analyte transmission efficiency by holding the analytes in the liquid-phase particle beam. But no evidence of adduct ions $(M+1$ or $M+18)$ was observed in any of the mass spectra, which retain a strict EI character. Therefore either this mechanism is not operational or the adduct ions are rapidly dissociated during flash vaporization from the hot ion source walls.

A related explanation for the ion abundance enhancements is the formation of relatively weak molecule-ammonium ion complexes in the mobile phase and the liquid phase of the spray. These ion-molecule aggregates are expected to form through hydrogen bonding interactions (for example, structure 1) or weak dipole-dipole interactions. They would be expected to increase the effective mass, increase the solubility, and reduce the vapor pressure of those analytes capable of forming complexes with ammonium ions. Thus the effect of ammonium acetate may be to reduce the vaporization of some analytes from the droplets of the spray and reduce subsequent analyte losses in the momentum separator.

All the analytes in the test mixture contain one or more functional groups capable of forming hydrogen bonds analogous to that shown in structure 1 . Some compounds without polar functional groups-for example, naphthalene and bis(perfluorophenyl)phenyl phosphine-are strongly vaporized and lost in the momentum separator and give 10-100 times weaker signals with the PB interface than when a GC inlet is used. After entry into the high vacuum of the ion source, these weak complexes must be rapidly dissipated during flash vaporization of the particles on the hot $\left(250^{\circ} \mathrm{C}\right)$ walls of the ion source.

$$
\mathrm{R}_{2} \mathrm{C}=\mathrm{O} \cdots \stackrel{+}{\mathrm{H}} \cdots \mathrm{NH}_{3}
$$

1

\section{Coeluting Compounds}

In addition to ammonium acetate, other substances produce enhanced ion abundances when they are present with analytes in the PB interface. This process was observed first when rotenone, propachlor, and caffeine were coinjected in flow injection analysis experiments and confirmed with coeluting substances in the HPLC/MS experiments. Therefore, as indicated previously, the enhancement factors in Table 1 for the three coeluting pairs (native and isotope-labeled) are combinations of two separate effects.

Experiments were conducted with gradient elution HPLC and ammonium acetate in the mobile phase to examine the effects of coeluting compounds on ion abundance and to determine whether this effect could be subdued by ammonium acetate. The test mixture was injected using system A (amounts injected shown in Table 1), and the ammonium acetate concentration in the reagent water solvent varied from 0 to $0.05 \mathrm{M}$. Figures 4-6 show the effects of ammunium acetate and the three coeluting native compounds on the integrated ion abundances of the corresponding stable isotopelabeled compounds. Each point in Figures 4-6 is the mean of two or more measurements, and repetitive measurements are within $5-15 \%$ of one another.

At zero ammonium acetate concentration and without coelution of the native compounds (lower three lines in Figures 4-6), caffeine- ${ }^{15} \mathrm{~N}_{2}$, benzidine- $\mathrm{D}_{8}$, and

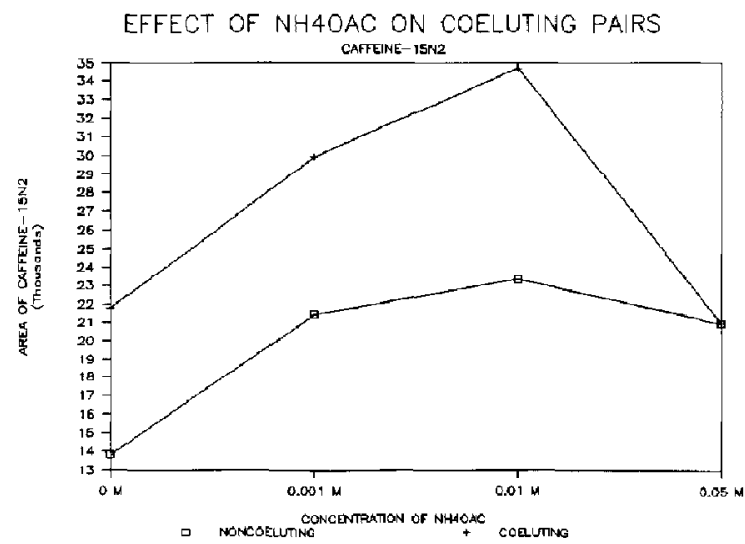

Figure 4. Effect of ammonium acetate and native caffeine on the integrated ion abundance of caffeine- ${ }^{-15} \mathrm{~N}_{2}$. 


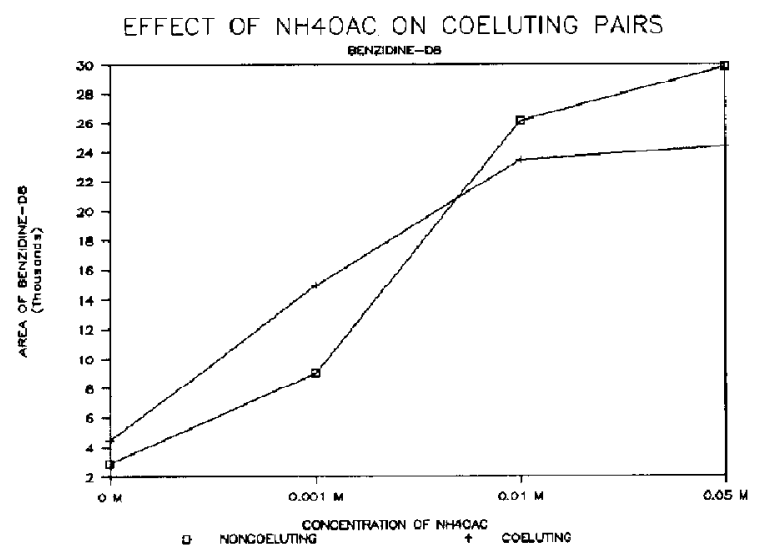

Figure 5. Effect of ammonium acetate and native benzidine on the integrated ion abundance of benzidine- $D_{8}$.

3, 3'-dichlorobenzidine- $D_{6}$ gave integrated abundances of $13,800,3000$, and 6800 , respectively. Inclusion of the coeluting native compounds at concentrations 1.75-4 times (Table 1) those of the labeled compounds but still without ammonium acetate caused the integrated abundances of the labeled compounds to increase to $21,900,4300$, and 23,800 (enhancement factors of 1.6 , 1.4 , and 3.5), respectively (upper three lines). As the concentration of ammonium acetate was increased at constant concentration of the coeluting pairs, the salt exerted a leveling effect on the integrated abundances at $0.05 \mathrm{M}$ for caffeine $-{ }^{15} \mathrm{~N}_{2}$, at about $0.008 \mathrm{M}$ for benzidine- $D_{8}$, and probably beyond $0.05 \mathrm{M}$ for $3,3^{\prime}$ dichlorobenzidine- $D_{6}$. Above an initial $0.05 \mathrm{M}$ ammunium acetate concentration in the reagent water, the momentum separator began to plug, and the experiment was terminated.

The processes operating to increase ion abundance with coeluting substances may result from the for-

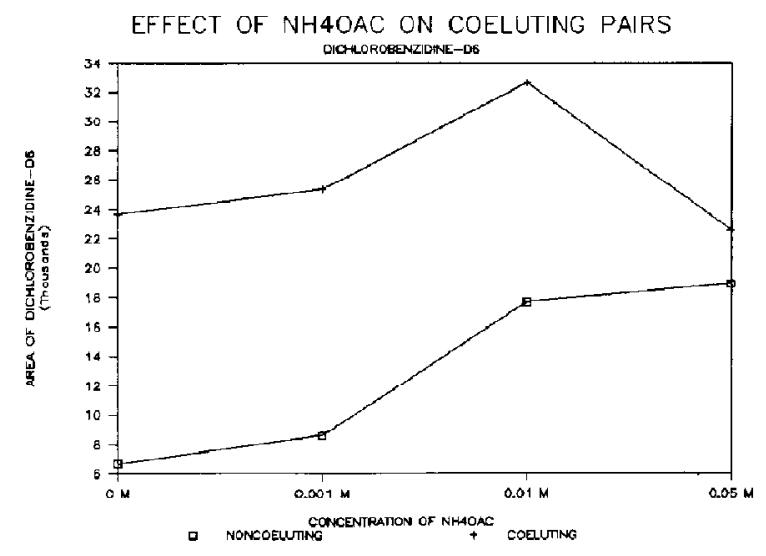

Figure 6. Effect of ammonium acetate and native $3,3^{\prime}$ dichlorobenzidine on the integrated ion abundance of $3,3^{\prime}$ dichlorobenzidine- $D_{6}$.

mation of molecular aggregates, probably through dipole-dipole interactions or hydrogen bonding, in the liquid phase. These clusters persist in the particles of the spray and act as PB carriers by increasing the effective mass and reducing the vapor pressure of those analytes. Thus vaporization of analytes is reduced, as are losses of analytes in the momentum separator. Ammonium acetate may exert a leveling effect by dissociating the clusters, but this effect is a complex function of the solvent composition, the molecular structures of the analytes, and the relative concentrations of the substances in the liquid phase.

A concentration calibration plot for $3,3^{\prime}$-dimethoxybenzidine (Figure 7) clearly illustrates the value of ammonium acetate in increasing analyte sensitivity and extending the instrument detection limit to lower levels. Similar calibration plots were observed for other compounds.

\section{DTMETHOXYBENZIDINE CALIBRATION CURVE}

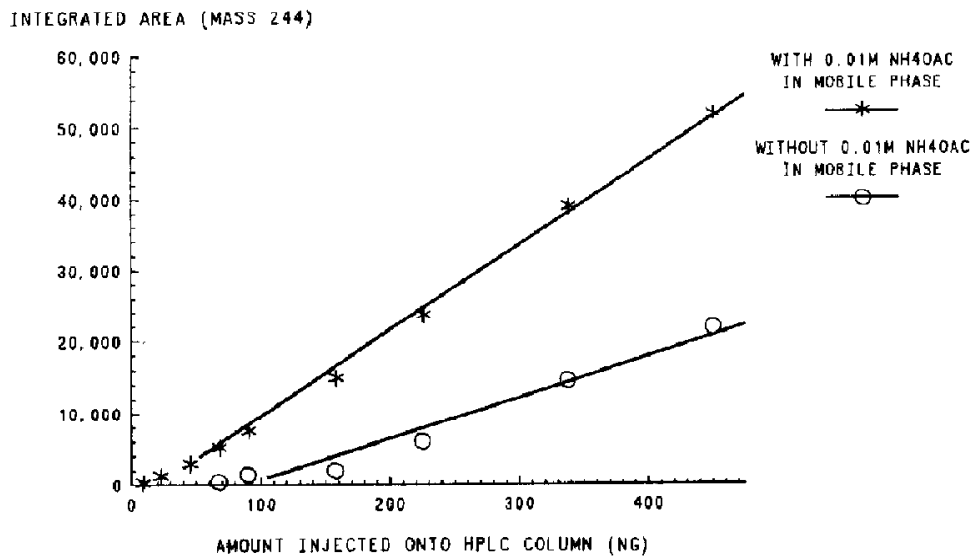

Figure 7. Concentration calibration plot for 3.3'-dimethoxybenzidine with and without ammonium acetate in the mobile phase. 


\section{Conclusion}

Ammonium acetate enhances ion abundances of polar analytes in $\mathrm{PB} / \mathrm{HPLC}$ MS by increasing the chromatographic efficiency of some compounds and by supporting a PB carrier process. The PB carrier process is also supported by neutral molecules capable of forming molecular clusters in solution. These processes may be used to enhance sensitivity in PB/HPLC/MS, but coeluting substances cause strong positive bias and nonlinear responses and may limit the applicability of $\mathrm{PB} / \mathrm{HPLC} / \mathrm{MS}$ for quantitative analyses. However, ammonium acetate may be capable of moderating the effect of coeluting substances.

\section{Acknowledgment}

We thank the Hewlett-Packard Corporation for the loan of the particle beam interface and the high-energy dynode detector.

\section{Disclaimer}

Mention of trade names or commercial products does not constitute endorsement or recommendation for use.

\section{References}

1. Willoughby, R. C.; Browner, R. F. Anal. Chem, 1984, 56, 2626-2631.

2. Willoughby, R. C.; Poeppel, F. Proceedings of the 36th ASMS Conference on Mass Spectrometry and Allied Topics; Denver, CO, May 24-29, 1987, p 289.

3. Apffel, A.; Nordman, R. Proceedings of the 36th ASMS Confer. ence on Mass Spectrometry and Allied Topics; San Francisco, CA, June 5 10, 1988, p 1318.

4. Vestal, M. L_; Fergusson, G. J. Anal. Chem. 1985, 57, 2373-2378.

5. Bellar, T. A.; Budde, W. L. Anal. Chem. 1988, 60, 2076-2083. 